PSYCHOMETRIKA-VOL. 32, NO. 3

SEPTEMBER, 1967

\title{
A NOTE ON A MATRIX CRITERION FOR UNIQUE COLORABILITY OF A SIGNED GRAPH*
}

\author{
Terry C. Gleason and Dorwin Cartwright \\ UNIVERSTTY OF MICHIGAN
}

\begin{abstract}
A signed graph, $S$, is colorable if its point set can be partitioned into subsets such that all positive lines join points of the same subset and all negative lines join points of different subsets. $S$ is uniquely colorable if there is only one such partition. Developed in this note is a new matrix, called the type matrix of $S$, which provides a classification of the way pairs of points are joined in $S$. Such a classification yields a criterion for colorability and unique colorability.
\end{abstract}

Much work has appeared in recent years exploring the properties of natural clusterings in structures of various sorts. One branch of this work has stemmed from the thinking of Heider [1946, 1958], who suggested certain rules that seem to govern the arrangement of positive and negative affective relationships among cognitive elements. Heider described certain structures as being "balanced" and postulated that these would be preferred over other structures which were said to be "imbalanced." This formulation, stated in terms of "elements" and "signed relationships," suggested to Cartwright and Harary [1956] that the theory of signed graphs could be a useful tool for achieving a more rigorous and a more general treatment of structural balance. A signed graph $S$ consists of a set of points $V(S)$ together with a prescribed subset of the collection of all lines (i.e., unordered pairs of distinct points), where each line is designated as either positive or negative. Cartwright and Harary were able to give a characterization of balanced signed graphs in what they called the "structure theorem": the point set of a balanced signed graph can be separated into two disjoint sets (one of which may be empty) such that positive lines join points of the same set and negative lines join points of different sets.

Signed graphs can, of course, be given a great variety of interpretations. Most research to date has interpreted points as elements of cognition (objects of perception or thinking) and signed lines as relationships between pairs of these elements (such as perceived liking and disliking or approval and disapproval). Illustrative studies employing this type of interpretation are those of Morrissette [1958], Rosenberg and Abelson [1960], Kuethe and

*The work reported here was supported by Grant MH 10834 from the National Institute of Mental Health. 
DeSoto [1964], Zajone and Burnstein [1965], and Feather [1964]. The possibility of interpreting points as people and signed lines as positive or negative interpersonal relationships has also been explored by such investigators as Newcomb [1961] and Davis [1963]. The hypothesis of a tendency toward balance under any interpretation predicts that the empirical entities under consideration will tend to form (at most) two elusters such that pairs of elements in the same cluster have only positive relationships and pairs from different elusters have only negative ones.

Davis [1967] has suggested that empirical phenomena, particularly sociometric structures, may tend to cluster in the manner of the structure theorem but that under some conditions the number of clusters may exceed two. This observation led him to a more general characterization of those signed graphs which can be partitioned into $n$ sets satisfying the condition of the structure theorem.

Cartwright and Harary [in press] have recently shown that the problem of partitioning the points of a signed graph in this manner can be related to the classical problem of coloring graphs. In order to place the problem in this broader context, they advanced the following definitions, which we employ in this paper. A signed graph $S$ is colorable if there exists a partition of the points of $S$ into subsets called color sets, such that points joined by a positive line are in the same subset and points joined by a negative line are in different subsets. A signed graph is uniquely colorable if there exists only one partition of the points into color sets.

As an illustration of these concepts consider the signed graphs $S_{1}$ and $S_{2}$ displayed in Fig. 1. The convention is adopted that solid lines have a positive sign and dashed lines have a negative sign. It can be seen that $S_{2}$ is colorable whereas $S_{1}$ is not. In fact, $S_{2}$ is uniquely colorable, with color sets $\left\{v_{1}, v_{2}, v_{3}\right\},\left\{v_{4}\right\},\left\{v_{5}, v_{6}\right\}$. If one were to interpret points as people and lines as affective relationships, then one might expect the people represented by $S_{2}$ to form three clusters corresponding to the color sets. On the other hand, there would seem to be no natural clustering of the people represented by $S_{1}$, since it has no color sets. Let us suppose, however, that the negative line joining $v_{1}$ and $v_{3}$ in $S_{1}$ were deleted. The resulting signed graph would then be colorable, but not uniquely so. Its point set could be partitioned in two ways: $\left\{v_{1}, v_{2}, v_{3}\right\},\left\{v_{4}\right\},\left\{v_{5}, v_{6}\right\}$ and $\left\{v_{1}, v_{2}, v_{3}\right\},\left\{v_{4}, v_{5}, v_{6}\right\}$, and either of these might serve as a basis for clustering.

It is desirable, when dealing with signed graphs that are large or eomplex, to have a mechanical means for deciding if a given one is colorable, and if so, whether it is uniquely colorable. In the remainder of this paper a matrix method is developed for answering such questions.

We begin by defining some relevant matrix concepts. For the rest of this paper let $S$ be a signed graph with $p$ points and $G$ the graph obtained from $S$ by ignoring the signs on its lines. The adjacency matrix $A=\left[a_{i j}\right]$ 


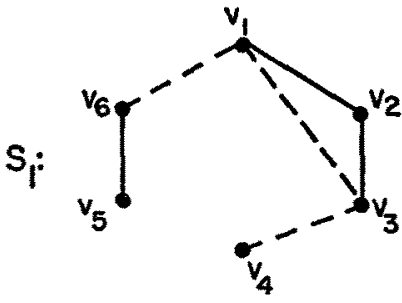

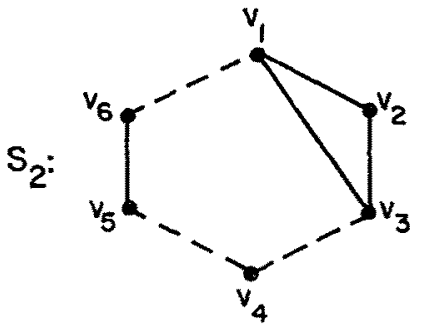

Figure 1

Two signed graphs illustrating the concept of colorability. $S_{1}$ is not colorable, whereas $S_{2}$ is uniquely colorable.

of $G$ is given by the following rule: number the points of $G$ from 1 to $p$ and construct a $p \times p$ matrix such that $a_{i j}=1$ if and only if there is a line joining $v_{i}$ and $v_{i}$ in $G$ and $a_{i j}=0$ otherwise. The reachability matrix $R=\left[r_{i j}\right]$ of $G$ is also a $p \times p$ matrix having a 1 in the $i, j$ entry if there is a path joining points $v_{i}$ and $v_{i}$ in $G$ and a 0 otherwise. It has been shown by Harary, Norman, and Cartwright [1965] that $R=(I+A)^{p-1} \#$ where $I$ is the identity matrix and $\#$ indicates that the arithmetic performed is boolean (i.e., $1+1=1$ ).

Let $S^{+}$be the spanning subgraph obtained by removing all the negative lines from $S$. Let $A^{+}$be the adjacency matrix of $S^{+}$and $R^{+}=\left(I+A^{+}\right)^{p-1} \#$. Similarly let $S^{-}$be the subgraph obtained by deleting all the positive lines from $S . A^{-}$will stand for the adjacency matrix of $S^{-}$. (Note that unlike some other usage, $A^{-}$will have only non-negative entries.) Finally let $M$ be a matrix given by the following expression.

$$
M=\left(R^{+} \cdot A^{-} \cdot R^{+}\right) \# .
$$

Lemma 1 . Let $M=\left[m_{i j}\right]$. Then $m_{i j}=1$ if and only if there is a sequence of lines in $S$ joining points $v_{i}$ and $v_{i}$ that contains exactly one negative line.

Proof: Let $m_{i i}=1$. Now $m_{i j}$ is of the form $\sum_{s} \sum_{k} r_{i k}^{+} \cdot a_{k h}^{-} \cdot r_{h i}^{+}=1$. Thus for some $k$ and some $h$ there exists a path in $S$ consisting of all positive lines joining the points $v_{i}$ and $v_{k}$, a negative line joining $v_{k}$ and $v_{h}$, and $a$ path consisting of all positive lines joining $v_{b}$ and $v_{i}$. Thus there is a sequence of lines joining $v_{i}$ and $v_{i}$ which contains exactly one negative line.

Now assume there is a sequence of lines joining points $v_{i}$ and $v_{i}$ containing exactly one negative line. Let the negative line join points $v_{k}$ and $v_{h}$. Then clearly $r_{i k}^{+}=1, a_{k h}^{-}=1$, and $r_{h i}^{+}=1$. Hence $m_{i j}=1$.

Q.E.D.

For the remainder of the discussion it will be helpful if we adopt the following conventions. A path in $S$ consisting of all positive lines will be called an all-positive path. A sequence of lines containing exactly one negative line will be called a I-negative sequence.

We require two lemmas on the colorability of signed graphs. The first originally appeared in Davis [1967], and both appear as Theorems 1 and 2 
in Cartwright and Harary [in press]. They are given here, in slightly altered form, without proof.

Lemma $2 . S$ is colorable if and only if $S$ has no cycle with exactly one negative line.

Lemma 3: Let $S$ be colorable. $S$ is uniquely colorable if and only if for every two points $v_{i}$ and $v_{i}$ in $S$ exactly one of the following holds:

(i) $v_{i}$ and $v_{i}$ are joined by an all-positive path,

(ii) $v_{i}$ and $v_{i}$ are joined by a 1 -negative sequence.

Let $T=\left[t_{i}\right]$ be a $p \times p$ matrix, called the type matrix of $S$, given by the following equation:

$$
T=[2]+R^{+}-2 M,
$$

where [2] stands for the $p \times p$ matrix with a 2 in every entry. Note that since the matrices $R^{+}$and $M$ have entries of only 0 and 1 , the largest value of $t_{i j}$ will occur when $r_{i i}^{+}=1$ and $m_{i j}=0$, in which case $t_{i j}=3$. The smallest value of $t_{i j}$ results when $r_{i j}^{+}=0$ and $m_{i j}=1$ for which $t_{i j}=0$. Hence for all $i$ and $j, 0 \leq t_{i j} \leq 3$.

Lemma 4. The following four statements characterize the entries $t_{i j}$ of $T$.

(i) $t_{i j}=0$ if and only if points $v_{i}$ and $v_{i}$ are joined by a 1-negative sequence and no all-positive path.

(ii) $t_{i j}=1$ if and only if points $v_{i}$ and $v_{i}$ are joined by a 1-negative sequence and by an all-positive path.

(iii) $t_{i i}=2$ if and only if points $v_{i}$ and $v_{i}$ are joined neither by a $1-$ negative sequence nor by an all-positive path.

(iv) $t_{i j}=3$ if and only if points $v_{i}$ and $v_{i}$ are joined by an all-positive path and by no 1-negative sequence.

The above statements can be summarized by the following table:

Value of $t_{i j}$ Joined by an all-pos. path Joined by a 1-neg. sequence

$\begin{array}{lll}3 & \text { yes } & \text { no } \\ 2 & \text { no } & \text { no } \\ 1 & \text { yes } & \text { yes } \\ 0 & \text { no } & \text { yes }\end{array}$

Proof of Lemma 4. By the definition of $R^{+}, r_{i j}^{+}=1$ if and only if there is. in $S$ an all-positive path joining $v_{i}$ and $v_{i}$. By Lemma $1, m_{i j}=1$ if and only if $v_{i}$ and $v_{i}$ are joined by a 1-negative sequence. These observations, together with the definition of $T$, lead immediately to statements (i)-(iv).

Q.E.D. 
Lemma 5. $S$ is colorable if and only if $T$ has no entry $t_{i i}=1$.

This is an immediate consequence of Lemmas 2 and 4 and the observation that an all-positive path joining two points together with a 1-negative sequence between those same points contains a cycle with exactly one negative line.

Sufficient background has now been developed to give a matrix criterion for unique colorability.

Theorem. $S$ is uniquely colorable if and only if $S$ is colorable and $T$ has no entries $t_{i i}=2$.

Proof: Assume $S$ is uniquely colorable. Then, by Lemma 3, for any two points $v_{i}$ and $v_{i}$ either (a) $v_{i}$ and $v_{i}$ are joined by an all-positive path, hence by Lemma 4 (iv) $t_{i i}=3$, or (b) $v_{i}$ and $v_{i}$ are joined by a 1 -negative sequence, hence by Lemma 4 (i) $t_{i j}=0$. Thus for all $i$ and all $j, t_{i j} \neq 1$, i.e., $S$ is colorable, and $t_{i j} \neq 2$.

Now assume $S$ is colorable and $t_{i j} \neq 2$ for all $i$ and all $j$. Then by Lemma 4 either $t_{i i}=3$ or $t_{i i}=0$. Hence by Lemma $3 S$ is uniquely colorable. Q.E.D.

As an illustration of the method developed above, Fig. 2 gives the type matrices for the signed graphs of Fig. 1. It is immediately evident that $S_{1}$ is not colorable since its type matrix, $T_{1}$, contains several 1's. The signed graph $S_{2}$, on the other hand, is uniquely colorable since its type matrix, $T_{2}$, contains only 0 's and 3's. Actually, the type matrices $T_{1}$ and $T_{2}$ give much more information than the existence of coloring in the signed graphs. We have in fact a classification of the way pairs of points in $S$ may be colored. For example, $t_{i i}=0$ indicates that the points $v_{i}$ and $v_{i}$ must be given different colors in all partitions of $V(S)$, whereas $t_{i}=3$ means $v_{i}$ and $v_{i}$ must be given the same color. An entry $t_{i i}=2$ indicates that $v_{i}$ and $v_{j}$ may be given the same or different colors, i.e., they may be placed in the same or different color sets in any partition of $V(S)$, whereas $t_{i j}=1$ means that no assignment of colors to $v_{i}$ and $v_{i}$ will satisfy the conditions of colorability.

$$
\mathrm{T}_{1}=\left[\begin{array}{llllll}
1 & 1 & 1 & 0 & 0 & 0 \\
1 & 1 & 1 & 0 & 0 & 0 \\
1 & 1 & 1 & 0 & 0 & 0 \\
0 & 0 & 0 & 3 & 2 & 2 \\
0 & 0 & 0 & 2 & 3 & 3 \\
0 & 0 & 0 & 2 & 3 & 3
\end{array}\right]
$$$$
T_{2}=\left[\begin{array}{llllll}
3 & 3 & 3 & 0 & 0 & 0 \\
3 & 3 & 3 & 0 & 0 & 0 \\
3 & 3 & 3 & 0 & 0 & 0 \\
0 & 0 & 0 & 3 & 0 & 0 \\
0 & 0 & 0 & 0 & 3 & 3 \\
0 & 0 & 0 & 0 & 3 & 3
\end{array}\right]
$$

Fraune 2 


\section{REFERENCES}

Cartwright, D. and Harary, F. Structural balance: A generalization of Heider's theory, Psychological Review, 1956, 63, 277-293.

Cartwright, D., and Harary, F. On the coloring of signed graphs. Elemente der Mathematik, (in press).

Davis, J. A. Structural balance, mechanical solidarity and interpersonal relations. American Journal of Sociology, 1963, 68, 444-462.

Davis, J. A. Clustering and structural balance in graphs. Human Relations, 1964, 20, 181-187.

Feather, N. T. A structural balance model of communication effects. Psychological Review, $1964,71,291-313$.

Harary, F., Norman, R. Z., and Cartwright, D. Structural models: an iniroduction to the theory of directed graphs. New York: Wiley, 1965.

Heider, F. Attitudes and cognitive organization. Journal of Psychology, 1946, 21, 107-112.

Heider, F. The Psychology of interpersonal relations. New York: Wiley, 1958.

Kuethe, J. L. and De Soto, C. B. Grouping and ordering schemata in competition. Psychonomic Science, 1964, 1, 115-116.

Morrissette, J. An experimental study of the theory of structural balance. Human Relations, $1958,11,239-254$.

Newcomb, T. M. The acquaintance process. New York: Holt, Rinehart, and Winston, 1961.

Rosenberg, M. J. and Abelson, R. P., An analysis of cognitive balancing. In C. I. Hovland and M. J. Rosenberg (Eds.), Attitude, organization and change. New Haven: Yale Univer. Press, 1960, 112-163.

Zajonc, R. B. and Burnstein, E. The learning of balanced and unbalanced social structures. Journal of Personality, 1965, 33, 153-163.

Manuscript received 9/9/66

Revised manuscript received $8 / 28 / 67$ 